\title{
A simple vaccination model with multiple endemic states
}

\author{
Christopher M. Kribs-Zaleta \\ Department of Mathematics \\ University of Texas at Arlington \\ Arlington, TX 76019-0408 \\ Jorge X. Velasco-Hernández \\ Departamento de Matemáticas \\ UAM-Iztapalapa \\ Apdo Postal 55-534, México, D.F. 09390
}

\begin{abstract}
A simple two-dimensional SIS model with vaccination exhibits a backward bifurcation for some parameter values. A two-population version of the model leads to the consideration of vaccination policies in paired border towns. The results of our mathematical analysis indicate that a vaccination campaign $\phi$ meant to reduce a disease's reproduction number $R(\phi)$ below one may fail to control the disease. If the aim is to prevent an epidemic outbreak, a large initial number of infective persons can cause a high endemicity level to arise rather suddenly even if the vaccine-reduced reproduction number is below threshold. If the aim is to eradicate an already established disease, bringing the vaccine-reduced reproduction number below one may not be sufficient to do so. The complete bifurcation analysis of the model in terms of the vaccine-reduced reproduction number is given, and some extensions are considered.
\end{abstract}

\section{Introduction}

Recently there has been interest in the analysis and prediction of consequences of public health strategies designed to control infectious diseases, particularly tuberculosis and AIDS. Attention has been given to vaccination and treatment policies both in terms of the different vaccine classes (all/nothing, leaky, VEI) and efficacy (e.g., [42], [3], [16], [17], [25]) and to application schedules and associated costs (e.g., [28], [29], [21], [22], [40], [2]).

The study of vaccination, treatment and associated behavioral changes related to disease transmission has been the subject of intense theoretical analysis. The literature on these topics is ample and in the references we list but a small subset of it. We want to underline, however, a few of those that directly relate to the approach we take in this work.

The application of a vaccination and treatment programs has the likely effect of inducing behavioral changes in those individuals subjected to it. In particular, in the case of HIV, risk behavior can combine with vaccination or treatment resulting in a possible harmful effect in terms of disease prevalence. Blower and McLean [4] have argued that a mass vaccination campaign can increase the severity of disease if the vaccine being applied resulted in only a $50 \%$ coverage and $60 \%$ efficacy. Velasco-Hernández and Hsieh [44] and Hsieh and Velasco-Hernández [30] confirmed this result in a theoretical mathematical model of disease transmission. In their case a too-large case treatment rate combined with lengthening of the infectious period could result in the increase of the treatment 

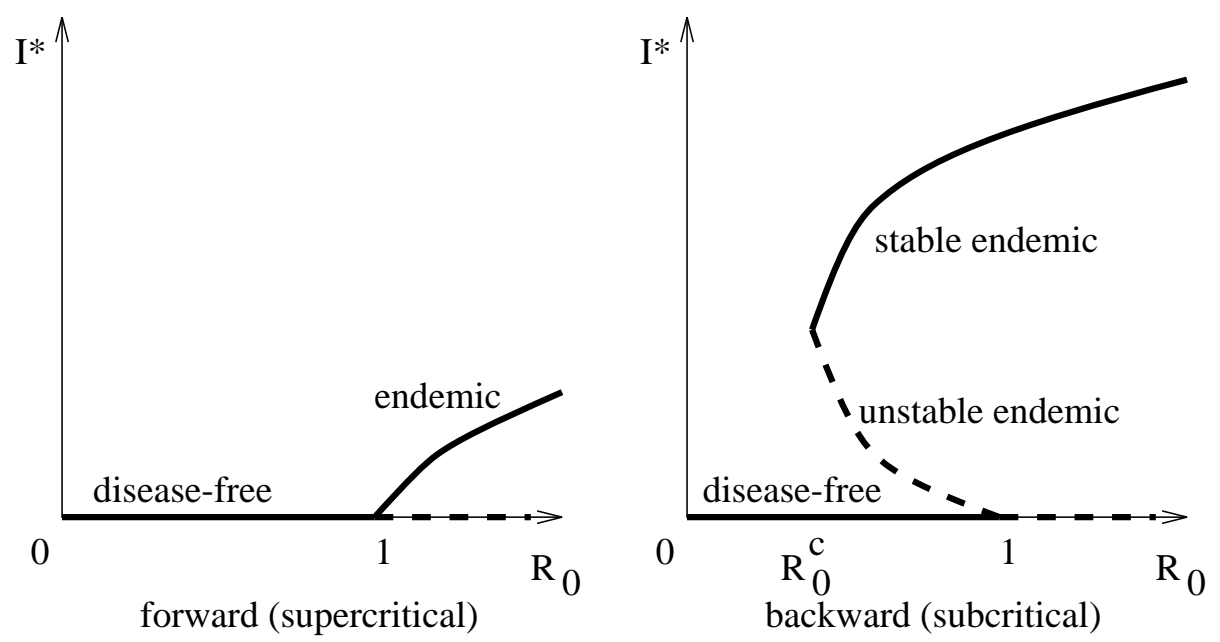

Figure 1: A comparison of the (a) forward and (b) backward bifurcations at $R_{0}=1$

reproduction number, that is, treatment would contribute to the spread of disease rather than to its elimination.

Of course, these are theoretical investigations on the plausible effects of vaccination and treatment programs. The models are rather simple, but nevertheless they give insight into some of the plausible consequences of public health policies.

A phenomenon of considerable interest recently in theoretical epidemiology is that of the existence of multiple steady-states and the associated population and epidemiological mechanisms that produce them. Mathematical models that give rise to multiple steady-states show bifurcation phenomena. A bifurcation in general is a set of parameter values at which an equilibrium, or fixed point, of the system being considered changes stability and/or appears/disappears. In epidemiology, bifurcation phenomena are associated with threshold parameters, the most common of which is the basic reproduction number, $R_{0} . R_{0}$ is a dimensionless quantity that represents the average number of secondary infections caused by an infective individual introduced into a pool of susceptibles. In the case of the simplest epidemiological models of the SIS and SIR types and a considerable number of generalizations (e.g., [38]), if $R_{0}<1$, the pathogen cannot successfully invade the host population, and dies out; if $R_{0}>1$, however, the pathogen can invade and successfully colonize hosts, therefore producing an epidemic outbreak that in many cases ends up in the establishment of an endemic disease in steady-state.

The mathematical description of this phenomenon involves a so-called transcritical bifurcation that brings about an exchange in stability between the disease-free equilibrium, which exists for all values of $R_{0}$, and an endemic equilibrium which only exists on one side of the bifurcation point. (On the other side, it has a negative value and is therefore outside the biologically feasible state space.) Prototypical $R_{0}$ threshold behavior features a "forward" bifurcation, in which the endemic equilibrium exists only for $R_{0}>1$, so that there is no possibility of an endemic state when $R_{0}<1$. In systems exhibiting a backward bifurcation, however, the endemic equilibrium exists for $R_{0}<1$, so that under certain initial conditions it is possible for an invasion to succeed, or for an established endemic state to persist, with $R_{0}<1$. See Figure 1 for an illustration.

The presence of a backward bifurcation has other important consequences for the population dynamics of infectious diseases. In a system with a forward bifurcation, if parameters change and cause $R_{0}$ to rise slightly above one, a small endemic state results; that is, the endemic level at equilibrium is a continuous function of $R_{0}$ ([38]). In a system with a backward bifurcation, the 


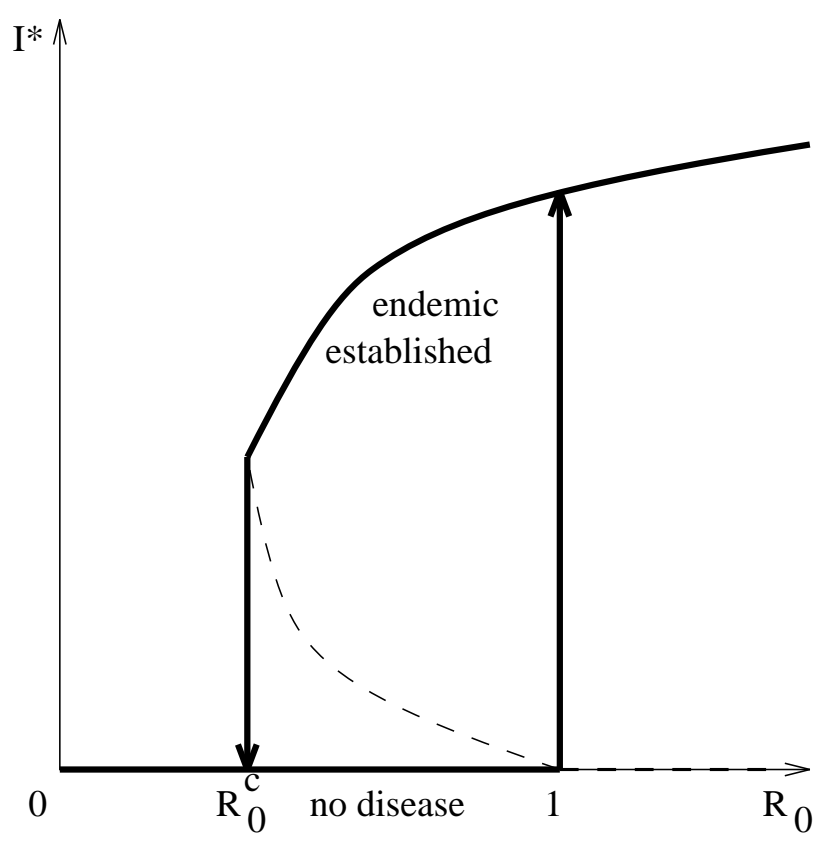

Figure 2: Backward bifurcation with hysteresis loop

endemic equilibrium that exists for $R_{0}$ just above one has a large infective population, so the result of $R_{0}$ rising above one would be a sudden and dramatic jump in the number of infectives. Moreover, reducing $R_{0}$ back below one would not eradicate the disease, if the infective population size is close to the endemic level at equilibrium: there will be two locally stable equilibrium points, one with no disease and other with a positive endemic level. In this case, in order to eradicate the disease, one must further reduce $R_{0}$ so far that it passes a so-called saddle-node bifurcation at $R_{0}^{c}<1$ (see Figure 2) and enters the region where no endemic equilibria exist, and the disease-free equilibrium is globally asymptotically stable.

This effect is known as hysteresis, and the system is said to have memory: that is, for $R_{0}$ between $R_{0}^{c}$ and 1 , one can tell whether $R_{0}$ was most recently less than $R_{0}^{c}$ or greater than 1 (barring a sudden significant invasion of infectives).

Models which exhibit backward bifurcations have been studied in an epidemiological context. Castillo-Chávez et al [8], Dushoff [12], Dushoff, Huang and Castillo-Chávez [13], Hadeler and Castillo-Chávez [19], Hadeler and van den Driessche [23], Huang, Cooke and Castillo-Chávez [31], and Kribs-Zaleta [34], [36] have all considered models that exhibit backward bifurcations. More recently, Hadeler and van den Driessche [23] reviewed such models and found that multi-group models with asymmetry between groups, or multiple interaction mechanisms, can cause backward bifurcations, and Dushoff, Huang and Castillo-Chávez [13] derived a criterion for determining the direction of the bifurcation at $R_{0}=1$. Finally, Kribs-Zaleta [37] used a more general approach to analyze bifurcations in epidemic models. Here it was also concluded that backward bifurcations tend to arise when the population is compartmentalized in other ways besides infectedness (typically by contact rates), and individuals can move between these compartments. Backward bifurcations have also been found in metapopulation models where the phenomenon has been associated with relatively high impact of migration on local patch dynamics [15].

As mentioned before, behavioral change has been the subject of mathematical analysis in models for the spread of infectious diseases as it relates to mixing patterns [33], isolation [32], treatment [44], [30], [45], and vaccination and education programs [19]. One of the main reasons for modeling 
these processes is to predict the effect of public health policies, particularly the implementation of treatment and vaccination campaigns. In this paper we concentrate on vaccination policies and vaccine-related parameters: vaccine coverage (rate at which susceptible individuals are immunized per unit time), average duration of immunity acquired by vaccine application (waning period), and the leakiness of the vaccine, that is, the percentage of susceptible individuals left unprotected even though vaccinated.

There are different types of vaccines (see, e.g., [14], [42]): some may give permanent immunity, while others offer only temporary protection; vaccines may not show $100 \%$ efficacy (leaky vaccines), and, finally, vaccine coverage may not be $100 \%$. There are other public health strategies that behave in a fashion similar to that of the vaccine, but instead of affecting the immune defenses on individuals, they affect behaviors that may impact (reduce or increase) disease transmission. These we call public education programs (see, e.g., [19]).

Mass vaccination as a control mechanism attempts to lower the degree of susceptibility of a healthy individual against a particular pathogenic agent. Since this decrease of susceptibility occurs in a population, the overall effect of mass vaccination is to decrease the proportion of contacts with infected individuals, giving rise to the concept of herd immunity. At the population level, therefore, one wishes to identify the critical vaccination rate necessary to reduce a certain threshold parameter below one so as to eradicate the disease or prevent infection (epidemic outbreak) [1], [19], [40], [2].

However, as noted above, in models that can exhibit multiple endemic equilibria, ensuring a below-threshold value for the bifurcation parameter is insufficient to ensure that a disease is wiped out [19], [44]. In particular, it has been seen that partially effective disease management programs may actually be worse than none at all.

Because systems with multiple endemic states tend to be relatively complex, it is often difficult to provide a complete mathematical analysis of them. In this paper we present as simple a system of this type as possible - a two-dimensional model that exhibits a backward bifurcation - along with a complete analysis of its behavior.

The system considered in Section 2 models an SIS disease with a vaccinated class in a constant size closed population with homogeneous mixing. The vaccination policy illustrated in the model is one for an all/nothing vaccine which is leaky and confers only temporary immunity. The choice of an SIS disease framework has been made for the sake of generality. Setting the cure rate to zero in our equations leaves all of our results unchanged for an SI disease model. Likewise, the immunity can be made permanent by setting a different parameter to zero, without changing any results.

In Section 3, we extend the system in two different ways. In Sections 3.1 and 3.2, the model expands to four dimensions in order to model two coexisting populations with different vaccination rates. This latter model was conceived to model a likely situation that may occur in neighboring border cities such as San Diego and Tijuana, or El Paso and Ciudad Juárez, whose populations interact on a daily basis while being subject to vaccination policies for the same disease which are conceivably very different. In the Mexican-US border example mentioned above, the vaccination policies of BCG and rubella are completely different in each country, and yet disease transmission takes place in either side of the border.

Section 3.3 considers a model which includes as special cases both the model of Section 2 and the model of primary focus in [19], in which some fraction of recovering infecteds pass directly into the vaccinated class, and vaccination is permanent.

Finally, we conclude in Section 4 with a discussion of the mathematical and biological consequences of our findings. 


\section{A vaccination model}

\subsection{Model formulation and $R_{0}$}

Let us now consider a model for an SIS disease where a vaccination program is in effect. We model new infections using the mass-action law, so that in general there are $\beta S I / N$ new infections in unit time, where $S, I$ and $N$ are the numbers of susceptibles, infectives and total population, respectively. We assume that the timescale of the disease is such that the overall change in population size $N$ is negligible as an invasion of disease takes its course [23], [26], or, equivalently, that the population has reached its carrying capacity [18]. Consequently the expressions for mass-action and standard incidence coincide (for a discussion of standard incidence, see [27]). We also assume a constant recovery rate $c$. The vaccine has the effect of reducing the infection rate by a factor of $\sigma$, so that $\sigma=0$ means the vaccine is completely effective in preventing infection, while $\sigma=1$ means that the vaccine is utterly ineffective. The susceptible population is vaccinated at a constant rate $\phi$, and the vaccine also wears off at a constant rate $\theta$. We allow for renewal of the population, however, via the birth/death rate $\mu$; all newborns come into the susceptible class. We have chosen to present an SIS model, appropriate for diseases such as pertussis and tuberculosis, to illustrate our results. However, there is no loss in mathematical generality if we assume the cure rate to be equal to zero, thus obtaining an SI model more suited for diseases as AIDS and many others.

We can now formulate this model, dividing the population into three classes - susceptibles $(S)$, infectives $(I)$ and vaccinated $(V)$ :

$$
\begin{gathered}
S^{\prime}=\mu N-\beta S I / N-(\mu+\phi) S+c I+\theta V \\
I^{\prime}=\beta(S+\sigma V) I / N-(\mu+c) I \\
V^{\prime}=\phi S-\sigma \beta V I / N-(\mu+\theta) V
\end{gathered}
$$

Since we assume the population size $N$ constant, we can reduce the size of the model by letting $S=N-I-V$. Now the model is

$$
\begin{aligned}
& I^{\prime}=(N-I-(1-\sigma) V) \beta I / N-(\mu+c) I \\
& V^{\prime}=\phi(N-I-V)-\sigma \beta V I / N-(\mu+\theta) V
\end{aligned}
$$

reminding the reader of our parameters, all nonnegative:

$\beta$ is the infectious contact rate (per person, in unit time);

$c$ is the recovery rate for the disease (assumed constant);

$\mu$ is the natural birth/death rate (unrelated to disease);

$N$ is the population size (assumed constant);

$\phi$ is the vaccination rate;

$\theta$ is the rate at which the vaccination wears off;

$\sigma$ measures the efficiency of the vaccine as a multiplier to the infection rate: $0=$ completely effective, $1=$ useless; we take $0 \leq \sigma \leq 1$.

We note that results on well-posedness found in Miller [39] guarantee the existence and uniqueness of solutions to all models discussed in this paper, and continuous dependence on parameters. Also, solutions remain within the state space $I \geq 0, V \geq 0, I+V \leq N$.

We can write the equilibrium conditions by setting the left-hand side of each differential equation equal to zero. We can solve the second one for the equilibrium value $V^{*}=\frac{\phi\left(N-I^{*}\right)}{\mu+\theta+\phi+\beta \sigma \frac{I^{*}}{N}}$. 
Substituting this expression into the first condition and simplifying, we get a cubic equation in $\frac{I^{*}}{N}$. Factoring out $\frac{I^{*}}{N}$, which yields the disease-free equilibrium $I^{*}=0, V^{*}=\phi N /(\mu+\theta+\phi)$, we are left with a quadratic $f(x)=A x^{2}+B x+C=0$, where $x \equiv I^{*} / N, A=-\beta \sigma, B=$ $\sigma(\beta-(\mu+c))-(\mu+\theta+\sigma \phi)$, and $C=(\mu+\theta+\phi)\left(1-\frac{\mu+c}{\beta}\right)-(1-\sigma) \phi$.

Let us first consider the disease-free equilibrium. We can calculate the basic reproductive number $R$ for the disease - which determines the local stability of this equilibrium - by means of either the Jacobian matrix, obtained from linearizing the right-hand side of (1), or the nextgeneration matrix operator [10], [11], obtained by considering equilibria as fixed points of a map. $R$ is the dominant eigenvalue of the latter matrix, and appears in the dominant eigenvalue of the Jacobian matrix as a factor $(R-1)$. The reproductive number in the absence of vaccination is calculated to be $R_{0}=\frac{\beta}{\mu+c}$. When vaccination is present, we obtain the threshold parameter or vaccine reproduction number

$$
R(\phi)=\frac{\beta}{\mu+c} \frac{\mu+\theta+\sigma \phi}{\mu+\theta+\phi}
$$

We write $R(\phi)$ to emphasize the role of the vaccination rate in controlling the spread of the disease. Note that $R(0)=R_{0}$, and that $R(\phi) \leq R_{0}$ for all $\phi \geq 0$ (since $\sigma \leq 1$ ). In particular, the second factor in $R(\phi)$ shows how much the vaccination reduces $R_{0}$.

The disease-free equilibrium $I^{*}=0, V^{*}=\phi N /(\mu+\theta+\phi)$ is locally stable if and only if $R(\phi)<1$. Furthermore, Lemma 1 below shows that the disease-free equilibrium is globally stable if $R_{0}<1$. The proof, given in the appendix, uses a priori estimates and inequalities. See Figure 3 at the end of Section 2.2 for an illustration.

Lemma 1. For the system (1), the disease-free equilibrium is globally asymptotically stable if $R(0)=R_{0}<1$.

\subsection{Endemic equilibria and bifurcations}

Let us begin our investigation of endemic equilibria for model (1) by considering two extreme special cases.

First suppose that $\sigma=1$, i.e., the vaccine is useless. This makes the equation for $I$ independent of $V$, and $R(\phi)$ reduces to $R_{0}$. Now if $R_{0}>1, I(t)$ asymptotically approaches $\left(1-\frac{1}{R_{0}}\right) N$. Consequently, $V(t)$ approaches $\phi N /\left[(\mu+\theta+\phi+\beta) R_{0}-\beta\right]$. Here we observe classical $R_{0}$ threshold behavior.

If instead we suppose that $\sigma=0$, so that the vaccine is completely effective, then we find one (stable) endemic equilibrium, which exists only for $R(\phi)>1$ :

$$
I^{*}=\left[1-\frac{1}{R_{0}}\left(1+\frac{\phi}{\mu+\theta}\right)\right] N, \quad V^{*}=\frac{\phi N}{R_{0}(\mu+\theta)}
$$

Now we wish to answer the general question of what endemic equilibria are possible for $0<\sigma<1$. We return to the equilibrium condition $f(x)=A x^{2}+B x+C=0$. Note that $A<0$, and that $C=(\mu+\theta+\sigma \phi)(1-1 / R(\phi))$, so that $C>0$ precisely when $R(\phi)>1$. Note also that $f(0)=C, f(1)=A+B+C=-(\mu+c)(\mu+\theta+\phi+\beta \sigma) / \beta<0$, and that $f$ 's vertex $x=-\frac{B}{2 A}<\frac{\beta \sigma}{2 \beta \sigma}=\frac{1}{2}<1$ lies to the left of $x=1$. Now an endemic equilibrium corresponds to a solution of $f(x)=0$ on the unit interval $[0,1]$. By examining the quadratic $f$, we can see that there is exactly one endemic equilibrium whenever $C>0$, there are precisely two whenever $C<0, B>0$ (i.e., $-\frac{B}{2 A}>0$ ) and $B^{2}-4 A C>0$, and there are none otherwise. (There is also a bifurcation point 
with $C<0, B>0$ and $B^{2}-4 A C=0$, at which there is precisely one, but we shall deal with this later.)

The two parameters we are most likely to be able to change as public policy makers are $\beta$ and $\phi$; hence we can write the conditions in terms of $\beta$ (see below). They can also be put in terms of $\phi$, but the expressions are more complicated, less enlightening, and lack the linear correlation with $R(\phi)$ which $\beta$ affords. In either case, the ways these conditions can be satisfied - a large infection rate and a low vaccination rate - are plausible reasons for enabling the disease to invade. (The condition involving the discriminant is quadratic in both $\beta$ and $\phi$, but can be simplified using the condition $B>0$. The two conditions $B>0$ and $B^{2}-4 A C>0$ cannot, however, be reduced to one.) We therefore rewrite these conditions as follows:

Lemma 2. For model (1), with $R(\phi)$ as defined above,

(i) When $R(\phi)>1$, there is precisely one endemic equilibrium.

(ii) When $R(\phi)<1$, there are precisely two endemic equilibria if $\beta>\mu+c+\phi+(\mu+\theta) / \sigma$ and $\beta>\mu+c-(\mu+\theta+\sigma \phi) / \sigma+(2 / \sigma) \sqrt{(\mu+c)(1-\sigma) \sigma \phi}$. Otherwise there are none.

Stability analysis is hindered here by the complexity of the expressions for the endemic equilibria, which we might normally like to plug into the system's characteristic equation (and by the absence of other convenient relations between equilibrium values). For this reason we will instead determine stability by looking at bifurcation points. As evidenced by Lemma 2 above, model (1) does have the usual (transcritical) bifurcation at $R(\phi)=1$. Under certain conditions, to be determined below, this becomes a "backward" (subcritical) bifurcation, so that endemic equilibria exist for $R(\phi)<1$ as well as for $R(\phi)>1$.

In this case, there is some critical value of $R(\phi)$ below 1 , where a pair of endemic equilibria are created at a second, saddle-node type bifurcation (see again Figure 1). We can find this bifurcation point by setting the discriminant $B^{2}-4 A C$ to zero. Solving this equation for $R(\phi)$ yields $R(\phi)=\frac{4 \beta \sigma(\mu+\theta+\sigma \phi)}{4 \beta \sigma(\mu+\theta+\sigma \phi)+B^{2}}$, although we might better identify the bifurcation point in terms of $\beta$ by rewriting $B^{2}-4 A C=0$ as $\beta=\mu+c-(\mu+\theta+\sigma \phi) / \sigma+(2 / \sigma) \sqrt{(\mu+c)(1-\sigma) \sigma \phi}$. It is also possible, albeit not as clean, to solve this equation for $\phi$.

We can verify analytically that these two bifurcations are the only ones exhibited by model (1). As noted in the introduction, a bifurcation is a point at which the stability of some equilibrium of the system changes, by the crossing of one or more eigenvalues of the Jacobian matrix to the right of the imaginary axis in the complex plane. We can therefore look for bifurcations of this system by substituting $\lambda=i \nu, \nu \in \mathbb{R}$, into the characteristic equation. In this case, given a Jacobian of the form $\left(\begin{array}{ll}a_{11} & a_{12} \\ a_{21} & a_{22}\end{array}\right)$, the characteristic equation reduces to $|J-i \nu I|=\left(a_{11} a_{22}-a_{12} a_{21}-\nu^{2}\right)-i \nu\left(a_{11}+\right.$ $\left.a_{22}\right)=0$. Therefore, setting the real and complex parts to zero, $\nu= \pm \sqrt{a_{11} a_{22}-a_{12} a_{21}}$, and in addition either $\nu=0$ or $a_{11}+a_{22}=0$. We can rewrite this as follows: either (i) $a_{11} a_{22}-a_{12} a_{21}=0$ and $\nu=0$, or (ii) $a_{11}+a_{22}=0$ and $a_{11} a_{22}-a_{12} a_{21}>0$.

For the disease-free equilibrium $I^{*}=0, V^{*}=\frac{\phi N}{\mu+\theta+\phi}$, this becomes (i) $R(\phi)=1, \nu=0$, (ii) $R(\phi)=1+\frac{\mu+\theta+\phi}{\mu+c}$ and $R(\phi)<1$. The first of these is the one we expect; the second yields no points since it involves a contradiction.

It is more difficult to do the same thing for the endemic equilibrium, just as solving the characteristic equation is. If we try it, we will discover that condition (i) yields two points: the same bifurcation point we found above (since it is where the disease-free and endemic equilibria cross), as well as a saddle-node bifurcation at $I^{*} / N=-B / 2 A, B^{2}-4 A C=0, B>0$. Condition (ii), which has $\nu \neq 0$, identifies Hopf bifurcations, which give rise to limit cycles. It is difficult to solve condition (ii) explicitly, but we can show that it yields no bifurcation points by eliminating the possibility of periodic solutions. 
To do so, we will apply the Bendixson-Dulac criterion (see [24], p. 67 and a useful extension in [6], p. 268). First we point out that the line $I=0$ is invariant under model (1), and thus not involved in any periodic solutions. Next we observe that for $V<\phi(N-I) /\left(\mu+\theta+\phi+\beta \sigma \frac{I}{N}\right), V^{\prime}>0$, so the line $V=0$ cannot be involved in any, either, except possibly at the corner $I=N, V=0$. However, if we take $R_{0}>1$, which by Lemma 1 we can safely do, then $I^{\prime}<0$ in a neighborhood of $I=N$, so trajectories cannot take us there, either. Now we can apply Bendixson-Dulac using the auxiliary function $1 / I V$ on any subset of the state space excluding the axes. We calculate the divergence of the product $1 / I V$ times the right-hand side of system (1) and get $-\beta / V N-\phi(N-I) / I V^{2}$, which is negative away from the axes. This shows that there are no periodic solutions (or phase polygons) strictly in the interior of the state space. However, our arguments above ruled out any periodic solutions (or phase polygons) involving the axes, so we can conclude that there are none at all in our state space.

Therefore model (1) has at most two bifurcations. We will use now two different but related approaches to examine them. (Note that in more complicated systems it will be even more difficult to identify bifurcations in the way we have just done, but the techniques below will work on any that we do find.)

We first recall the criterion presented by Dushoff, Huang, and Castillo-Chávez [13] for determining the sign of the bifurcation at $R(\phi)=1$. This criterion examines the stability of the disease-free equilibrium along the eigenvector corresponding to the dominant eigenvalue, whose real part is zero at $R(\phi)=1$. In order to calculate the criterion, we must write our system in the form $u^{\prime}=H(u) \cdot\left(u-u^{*}\right)$, where $u^{*}$ is the disease-free equilibrium. The criterion $h$ is then calculated by

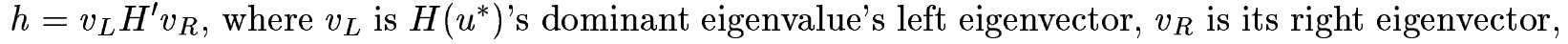
and

$$
H^{\prime}=\left.\frac{d}{d \alpha} H\left(u^{*}+\alpha v_{R}\right)\right|_{\alpha=0}
$$

If $h>0$, then the bifurcation at $R(\phi)=1$ is backward; if $h<0$, then it is forward.

We can write system (1) in this form by making $u=\left[\begin{array}{ll}I & V\end{array}\right]^{T}$, so that

$$
H(u)=\left(\begin{array}{cc}
-(\mu+c)+\frac{\beta}{N}(N-I-V(1-\sigma)) & 0 \\
-\phi\left(1+\frac{\beta \sigma}{\mu+\theta+\phi}\right) & -\left(\mu+\theta+\phi+\beta \sigma \frac{I}{N}\right)
\end{array}\right)
$$

The dominant eigenvalue of $H\left(u^{*}\right)$ is $(\mu+c)(R(\phi)-1)$, and we can calculate its associated eigenvectors, $v_{L}=\left[\begin{array}{ll}1 & 0\end{array}\right]$ and $v_{R}=\left[\begin{array}{ll}\frac{(\mu+\theta+\phi)^{2}}{\phi(\mu+\theta+\phi+\beta \sigma)} & -1\end{array}\right]^{T}$ (with the sign chosen to point into the state space). $H\left(u+\alpha v_{R}\right)$ is linear in $\alpha$, so we can calculate $H^{\prime}$ simply and find, after multiplying, that

$$
h=\frac{\beta}{N}\left[(1-\sigma)-\frac{(\mu+\theta+\phi)^{2}}{\phi(\mu+\theta+\phi+\beta \sigma)}\right] \frac{(\mu+\theta+\phi)^{2}}{\phi(\mu+\theta+\phi+\beta \sigma)}
$$

Thus, according to this criterion, in order to have a backward bifurcation at $R(\phi)=1$, with $h>0$, the term in brackets must be positive:

$$
(1-\sigma)-\frac{(\mu+\theta+\phi)^{2}}{\phi(\mu+\theta+\phi+\beta \sigma)}>0
$$

Note that this is only possible if $\sigma<1$, which agrees with our observation that the vaccine must have some effect in order to enable a backward bifurcation. 
Before we interpret this condition, let us consider the more general approach of Kribs-Zaleta [37]. For each bifurcation, we shall first reduce the dimension of the system to consider only the center manifold at the bifurcation point, a subset of the state space which acts as an attractor in other words, all solutions approach this manifold (in this case, a curve), and it is on the manifold that the interesting dynamics occur. For more background, see [7] and references in [37].

We compute [37] model (1)'s center manifold at $R(\phi)=1$ and the disease-free equilibrium as

$$
y=\frac{\beta}{N}(1-\sigma)\left[\frac{\phi(\mu+\theta+\phi+\beta \sigma)-(\mu+\theta+\phi)^{2}}{\phi(\mu+\theta+\phi+\beta \sigma)(\mu+\theta+\phi)}\right] x^{2}-\frac{\mu+c}{\mu+\theta+\phi} x p+\Gamma
$$

up to second-order terms, where $x \equiv-\frac{\phi(\mu+\theta+\phi+\beta \sigma)}{(\mu+\theta+\phi)^{2}} I, y \equiv \frac{\phi(\mu+\theta+\phi+\beta \sigma)}{(\mu+\theta+\phi)^{2}} I+V-V^{*}$, the bifurcation parameter $p \equiv R_{0}-1$, and $\Gamma$ represents all terms of order three in $x$ and $p$.

We now consider the dynamics on the center manifold, where we can write:

$$
x^{\prime}=(\mu+c) p x-\frac{\beta}{N}\left[(1-\sigma)-\frac{(\mu+\theta+\phi)^{2}}{\phi(\mu+\theta+\phi+\beta \sigma)}\right] x^{2}-\frac{\beta}{N}(1-\sigma) \frac{\mu+c}{\mu+\theta+\phi} x^{2} p+O\left(x^{3}\right)
$$

We compare this expression with the canonical form, or normal form, for a generic forward transcritical bifurcation: $x^{\prime}=p x-x^{2}+O\left(x^{3}\right)$. We see that the bifurcation at $R(\phi)=1$ is indeed transcritical iff $\tilde{h} \equiv(1-\sigma)-\frac{(\mu+\theta+\phi)^{2}}{\phi(\mu+\theta+\phi+\beta \sigma)} \neq 0$, and that the bifurcation is forward in $x$ - and therefore backward in $I$ - iff $\tilde{h}>0$, in agreement with the criterion of Dushoff, Huang and Castillo-Chávez above. (A linear transformation can remove the $x^{2} p$ term.)

This approach also allows us to verify the saddle-node bifurcation that exists when the transcritical bifurcation above is backward. We identify the point as $\left\{B^{2}-4 A C=0, B>0, C<0\right\}$ or $R(\phi)=\frac{4 \beta \sigma(\mu+\theta+\sigma \phi)}{4 \beta \sigma(\mu+\theta+\sigma \phi)+B^{2}}$, based on the equilibrium conditions we derived earlier. If we now define our bifurcation parameter $p^{2}=B^{2}-4 A C$ and let

$$
x \equiv \frac{-\frac{\sigma}{1-\sigma}[B+2(\mu+\theta+\phi)]\left(I-I^{*}\right)+B\left(V-V^{*}\right)}{B+\sigma[B+2(\mu+\theta+\phi)]}, y \equiv \frac{\frac{1}{1-\sigma}\left(I-I^{*}\right)+\left(V-V^{*}\right)}{B+\sigma[B+2(\mu+\theta+\phi)]}
$$

where $I^{*}=\frac{B N}{2 \beta \sigma}$ and $V^{*}=\frac{\phi N}{\beta \sigma} \cdot \frac{2 \beta \sigma-B}{B+2(\mu+\theta+\phi)}$, then we can find the center manifold in terms of $x$ and $p$, on which the dynamics are

$$
x^{\prime}=-\frac{B N}{4 \beta \sigma(1-\sigma)(B+\sigma[B+2(\mu+\theta+\phi)])} p+\frac{\beta}{N} \sigma(1-\sigma) \frac{B}{B+\sigma[B+2(\mu+\theta+\phi)]} x^{2}+O(x p)+O\left(p^{2}\right)+\Gamma .
$$

The normal form for a saddle-node bifurcation is $x^{\prime}=p \pm x^{2}+\Gamma$, and we can see that the expression above fits this form (again a linear transformation can eliminate the $O(x p)$ and $O\left(p^{2}\right)$ terms).

This approach also shows us that the stability of these "extra" endemic equilibria is indeed as we expected: for example, the forward transcritical bifurcation $x^{\prime}=p x-x^{2}$ has $x^{*}=0$ stable for $p<0$ and unstable for $p>0$, and vice versa for the equilibrium given locally by $x^{*}=p$. Since, for the transcritical bifurcation in model (1), I and $x$ differ in sign (see the definition of $x$ above), this means that when the bifurcation at $R(\phi)=1$ is backward in $I, I^{*}=0$ is stable for $R(\phi)<1$ $(p<0)$ and unstable for $R(\phi)>1(p>0)$, and, more importantly, vice versa for the endemic equilibrium which enters the state space through that bifurcation and exists for $R(\phi)<1$. The saddle-node bifurcation gives us similar information about the two endemic equilibria which meet there, and this local picture of (local) stability extends to a global picture of (local) stability - 
i.e., proves that the stability claimed in Figure 1 is accurate - unless there is another bifurcation in between, at which an eigenvalue of the characteristic equation crosses the imaginary axis (real part zero). However, we have seen above that there are no such other bifurcations.

Now note that we can easily solve the condition $\tilde{h}>0$ for $\beta$ : $\beta>\frac{(\mu+\theta+\phi)(\mu+\theta+\sigma \phi)}{\sigma(1-\sigma) \phi}$. From this we see, not surprisingly, that a sufficiently high contact rate in the presence of a partially effective vaccine will cause a backward bifurcation. The condition cannot be expressed so simply in terms of any of the other parameters; it is worth noting that the recovery rate $c$ does not appear, although of course it does appear in the equation $R(\phi)=1$. Since we know the condition cannot be satisfied for $\sigma=0$ or $\sigma=1$, there must be a certain range of effectiveness (i.e., of $\sigma$ ) for which a backward bifurcation will occur; likewise, for $\phi$ near 0 or $\phi$ sufficiently large, we see the condition cannot be met, so there is a range of vaccination rates which will allow it to happen.

We may be concerned that our criterion for the existence of a backward bifurcation in model (1) does not appear to match Lemma 2's three conditions for the existence of two endemic equilibria: $R(\phi)<1, B>0, B^{2}-4 A C>0$. However, a backward bifurcation only guarantees two endemic equilibria when parameters fall in the range $R^{c}(\phi)<R(\phi)<1$. The first and last of the above trio of conditions ensure that the parameters fall within this range; the first two conditions together imply $\tilde{h}>0$.

Now we can put the pieces together to form a complete picture of the model's behavior. We may divide the parameter space into three regions: (1) $R_{0}<1$, in which case, as shown in Lemma 1 , the disease-free equilibrium is globally stable; $(2) R(\phi)<1<R_{0}$, which is most of interest from a control perspective; and (3) $R(\phi)>1$, in which the vaccination program is not effective enough to bring the disease's reproductive number down, and the unique endemic equilibrium remains the only attractor. (Note that Poincaré-Bendixson applies since this is a two-dimensional system.)

In case (2), vaccination makes the disease-free equilibrium locally stable, but there remain two possibilities for global behavior: either the disease-free equilibrium is globally stable, or it competes with a locally stable endemic equilibrium. We can state the criteria for determining the global behavior most easily in terms of $\beta$. Let us define the following quantities; their individual significances are given at right.

$$
\begin{array}{ll}
\beta_{0}^{\prime} \equiv \mu+c & \beta<\beta_{0}^{\prime} \Leftrightarrow R_{0}<1 \text { (disease dies out regardless of vacc. program) } \\
\beta_{0} \equiv(\mu+c) \frac{\mu+\theta+\phi}{\mu+\theta+\sigma \phi} & \beta<\beta_{0} \Leftrightarrow R(\phi)<1 \text { (DFEQ a local attractor) } \\
\beta_{a} \equiv \mu+c+\frac{\mu+\theta+\sigma \phi}{\sigma} & \beta>\beta_{a} \Leftrightarrow B>0 \text { (ENEQs which may exist when } R(\phi)<1 \\
\beta_{b} \equiv \mu+c-\frac{\mu+\theta+\sigma \phi}{\sigma} & \beta>\beta_{b} \Leftrightarrow B^{2}-4 A C>0\left(R(\phi)>R^{c}(\phi)\right. \\
\quad+\frac{2}{\sigma} \sqrt{(\mu+c) \sigma(1-\sigma) \phi} & \\
\beta_{c} \equiv \frac{(\mu+\theta+\phi)(\mu+\theta+\sigma \phi)}{\sigma(1-\sigma) \phi} & \beta>\beta_{c} \Leftrightarrow \tilde{h}>0 \text { (backward be in state space) }
\end{array}
$$

We can see that $\beta_{0}^{\prime}<\beta_{0}$ and $\beta_{0}^{\prime}<\beta_{a}$, and we can show that either (a) $\beta_{c}<\beta_{a}<\beta_{b}<\beta_{0}$ or (b) $\beta_{b}<\beta_{0}<\beta_{a}<\beta_{c}$ (or all four are equal; this is why the endemic equilibrium conditions cannot be reduced). If $R(\phi)<1<R_{0}$, then we have $\beta_{0}^{\prime}<\beta<\beta_{0}$. If (a) holds, it is possible to have endemic equilibria if $\beta_{b}<\beta<\beta_{0}$ (note this implies $R(\phi)<1$ ); if (b) holds, then $R(\phi)<1\left(\beta<\beta_{0}\right)$ precludes the existence of endemic equilibria $\left(\beta>\max \left(\beta_{a}, \beta_{b}\right)\right)$. Inequality (a) can be rewritten more simply as $(\mu+\theta+\sigma \phi)^{2}<(\mu+c) \sigma(1-\sigma) \phi$. We can rewrite Lemma 2(ii)'s conditions as $\max \left(\beta_{a}, \beta_{b}\right)<\beta<\beta_{0}$. Thus, finally, we conclude that

Theorem 1. For model (1), with $R(\phi), \beta_{0}, \beta_{b}$ as defined above,

(i) If $(\mu+\theta+\sigma \phi)^{2}<(\mu+c) \sigma(1-\sigma) \phi$ and $\beta_{b}<\beta<\beta_{0}$, then two endemic equilibria exist, one of which is locally stable and competes with the locally stable disease-free equilibrium; 

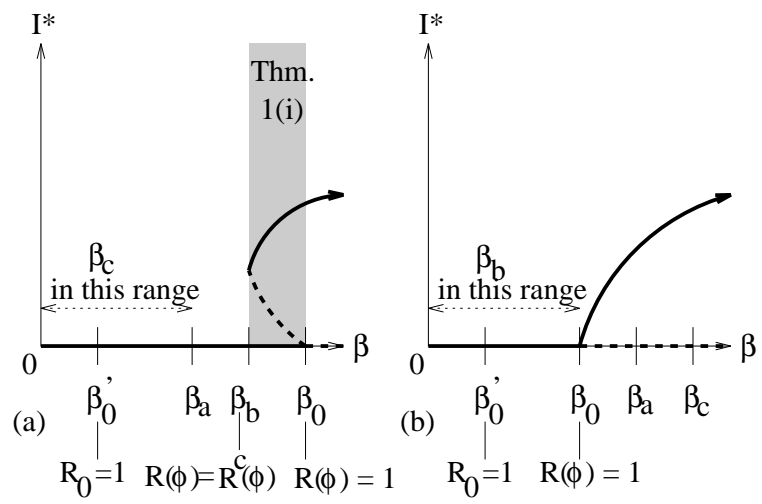

Figure 3: Bifurcation diagrams for model (1) in the two cases

(a) $\beta_{c}<\beta_{a}<\beta_{b}<\beta_{0}$ and (b) $\beta_{b}<\beta_{0}<\beta_{a}<\beta_{c}$

(ii) otherwise, the disease-free equilibrium is the unique attractor when $R(\phi)<1$.

Note that the backward bifurcation exists whenever $\beta>\beta_{c}$, but if parameter values make $R(\phi)>1\left(\beta>\beta_{0}\right)$ or $R(\phi)<R^{c}(\phi)\left(\beta<\beta_{b}\right)$, we will not observe its effect on system behavior. See Figure 3 for an illustration.

We can interpret biologically the endemic equilibrium which exists under the backward bifurcation as follows. Infected individuals arise from two sources: those susceptibles that get infected and those vaccinated that get infected. Under certain particular but nonetheless ample circumstances, migration into the infected class is large even though $R(\phi)<1$ : for a range of values of vaccination and infection rates, involving comparatively high infection and recovery rates, susceptibles are more likely to be infected than vaccinated, thus increasing the flow into the infected class. This promotes locally the existence of an endemic equilibrium for below-threshold values of the reproduction number. The above effect is valid only for population densities close to this endemic equilibrium. When initial conditions involve a sufficiently low number of infectives, it is not possible to sustain the exchange of susceptibles and infecteds necessary to establish an endemic state.

\section{Extensions}

In this section we shall consider two extensions of the model considered in the last section; results prove similar to those obtained above.

\subsection{A two-group model}

Now suppose we have two separate but interacting populations, such as those of border cities like San Diego and Tijuana, El Paso and Ciudad Juárez, or Laredo and Nuevo Laredo. A disease may be in circulation, and transmitted, among members of each population, as well as between populations. In this case, the two groups may have different values for model parameters, especially those dealing with vaccination. Suppose we allow $\mu$ (the natural birth/death rate) and $c$ (the recovery rate) to be the same for the two populations. We can now formulate a four-dimensional model of this situation by connecting two copies of our two-dimensional model (with $S_{1}$ and $S_{2}$ eliminated): 


$$
\begin{aligned}
& I_{1}^{\prime}=\left(N_{1}-I_{1}-\left(1-\sigma_{1}\right) V_{1}\right)\left(\beta_{1} I_{1}+\beta_{2} I_{2}\right) / N-(\mu+c) I_{1} \\
& V_{1}^{\prime}=\phi_{1}\left(N_{1}-I_{1}-V_{1}\right)-\sigma_{1} V_{1}\left(\beta_{1} I_{1}+\beta_{2} I_{2}\right) / N-\left(\mu+\theta_{1}\right) V_{1} \\
& I_{2}^{\prime}=\left(N_{2}-I_{2}-\left(1-\sigma_{2}\right) V_{2}\right)\left(\beta_{1} I_{1}+\beta_{2} I_{2}\right) / N-(\mu+c) I_{2} \\
& V_{2}^{\prime}=\phi_{2}\left(N_{2}-I_{2}-V_{2}\right)-\sigma_{2} V_{2}\left(\beta_{1} I_{1}+\beta_{2} I_{2}\right) / N-\left(\mu+\theta_{2}\right) V_{2}
\end{aligned}
$$

where $N_{1}$ and $N_{2}$ are the sizes of the two groups, assumed constants, and $N=N_{1}+N_{2}$. Note that the only interaction between populations is in the term representing new infections.

The equilibrium conditions are now too complicated to obtain a simple closed-form solution; however, we can see that the disease-free equilibrium $\left(0, \frac{\phi_{1} N_{1}}{\mu+\theta_{1}+\phi_{1}}, 0, \frac{\phi_{2} N_{2}}{\mu+\theta_{2}+\phi_{2}}\right)$ always exists. If we define the marginal vaccine reproduction numbers

$$
R_{1}\left(\phi_{1}\right)=\frac{\beta_{1}}{\mu+c} \frac{\mu+\theta_{1}+\sigma_{1} \phi_{1}}{\mu+\theta_{1}+\phi_{1}} \text { and } \mathrm{R}_{2}\left(\phi_{2}\right)=\frac{\beta_{2}}{\mu+\mathrm{c}} \frac{\mu+\theta_{2}+\sigma_{2} \phi_{2}}{\mu+\theta_{2}+\phi_{2}},
$$

analogous to $R(\phi)$ for the two-dimensional model, then local stability analysis of the disease-free equilibrium for the four-dimensional model yields the overall vaccine reproduction number

$$
R(\Phi)=R_{1}\left(\phi_{1}\right) \frac{N_{1}}{N}+R_{2}\left(\phi_{2}\right) \frac{N_{2}}{N},
$$

a weighted average of the two individual populations' $R(\phi)$ 's, where $\Phi=\left(\phi_{1}, \phi_{2}\right)$. Note that $R(0,0)=R_{0}$, the basic reproductive number for the disease (in the absence of any vaccination).

We can easily obtain the following result analogous to Lemma 1 (proof in appendix):

Theorem 2. For system (2), the disease-free equilibrium is globally asymptotically stable if

$$
R_{0}=\frac{\beta_{1}}{(\mu+c)} \frac{N_{1}}{N}+\frac{\beta_{2}}{(\mu+c)} \frac{N_{2}}{N}<1 .
$$

Note again that this does not preclude the stability of endemic equilibria which may exist for $R(\Phi)<1$, as they should occur for parameter values which do not satisfy the theorem hypothesis. Also, our interpretation is again that the vaccination extends the local (not necessarily global) stability of the disease-free equilibrium.

\subsection{Endemic equilibria of the two-group model}

We now turn to the existence of endemic equilibria. We can solve two of the equilibrium conditions for $V_{1}^{*}$ and $V_{2}^{*}$ in terms of $I_{1}^{*}$ and $I_{2}^{*}$ :

$$
V_{1}^{*}=\frac{\phi_{1}\left(N_{1}-I_{1}^{*}\right)}{\mu+\theta_{1}+\phi_{1}+\sigma_{1}\left(\frac{\beta_{1} I_{1}^{*}+\beta_{2} I_{2}^{*}}{N}\right)}, \quad V_{2}^{*}=\frac{\phi_{2}\left(N_{2}-I_{2}^{*}\right)}{\mu+\theta_{2}+\phi_{2}+\sigma_{2}\left(\frac{\beta_{1} I_{1}^{*}+\beta_{2} I_{2}^{*}}{N}\right)}
$$

The remaining conditions can thus be reduced to cubic equations of the form $h_{1}(x, y)=0$ and $h_{2}(x, y)=0$, where $x=I_{1}^{*} / N, y=I_{2}^{*} / N$. Any equilibria must then correspond to intersections of the zero contours of $h_{1}$ and $h_{2}$ within the domain $\left[0, N_{1} / N\right] \times\left[0, N_{2} / N\right]$. By analyzing these functions' zero contours, we can show the following (see appendix for proof).

Theorem 3. For model (2), with $R(\Phi)$ as defined above,

(i) for $R(\Phi)<1$, there is an even number of endemic equilibria;

(ii) for $R(\Phi)>1$, there is an odd number of endemic equilibria; 
(iii) for $R(\Phi)<1$, either of the conditions $\beta_{1}>\max \left(\beta_{1 a}, \beta_{1 b}\right), \beta_{2}>\max \left(\beta_{2 a}, \beta_{2 b}\right)$ is sufficient (but not necessary) to cause two endemic equilibria,

where $\beta_{1 a} \equiv \frac{N}{N_{1}}\left(\mu+c+\frac{\mu+\theta_{1}+\sigma_{1} \phi_{1}}{\sigma_{1}}\right), \beta_{1 b} \equiv \frac{N}{N_{1}}\left(\mu+c-\frac{\mu+\theta_{1}+\sigma_{1} \phi_{1}}{\sigma_{1}}+\frac{2}{\sigma_{1}} \sqrt{(\mu+c) \sigma_{1}\left(1-\sigma_{1}\right) \phi_{1}}\right)$, and $\beta_{2 a}$ and $\beta_{2 b}$ are defined similarly.

Furthermore, numerical results appear to indicate that the number of endemic equilibria is never more than two.

These results are a natural extension of those we obtained for model (1); clause (iii) yields an important consequence of the coupling of these two populations: each of the two is capable, under certain conditions, of bringing a stable endemic equilibrium into existence for $R(\Phi)<1$ regardless of how much effort the other population puts into vaccination and reducing its contact rate.

\subsection{From infection to immunity}

The model of primary focus in Hadeler and Castillo-Chávez [19] bears several structural similarities to the one studied in Section 2; the main difference is that vaccination is considered permanent $(\theta=0)$, and some fraction of recovering infecteds pass directly into this class; that is, it assumes that treatment and vaccination induce permanent immunity against the disease. In this sense, we could think of the model as an SIR model, or consider instead of vaccination and treatment an education program that changes behavior, effectively inducing immunity to disease transmission. We can easily extend system (1) to include this latter feature, introducing a parameter $\gamma, 0 \leq \gamma \leq 1$ [19], to denote the proportion of infecteds who pass directly into the vaccinated class upon recovery.

After reducing the model to two dimensions, as before, the only difference is a term $c \gamma I$ in the equation for $V^{\prime}$ :

$$
\begin{aligned}
& I^{\prime}=(N-I-(1-\sigma) V) \beta I / N-(\mu+c) I \\
& V^{\prime}=\phi(N-I-V)+c \gamma I-\sigma \beta V I / N-(\mu+\theta) V
\end{aligned}
$$

The analysis of this model parallels that given in Section 2, with only a few small differences. The disease-free equilibrium and expression for $R(\phi)$ are unchanged, but the analogues of $\beta_{a}, \beta_{b}$ and $\beta_{c}$ are:

$$
\begin{aligned}
& \beta_{\gamma a} \equiv \mu+c+\frac{\mu+\theta+\sigma \phi}{\sigma}+\frac{(1-\sigma) c \gamma}{\sigma} \\
& \beta_{\gamma b} \equiv \mu+c+\frac{(1-\sigma) c \gamma}{\sigma}-\frac{\mu+\theta+\sigma \phi}{\sigma}+\frac{2}{\sigma} \sqrt{(\mu+c) \sigma(1-\sigma) \phi-c \gamma(1-\sigma)(\mu+\theta+\sigma \phi)} \\
& \beta_{\gamma c} \equiv \frac{(\mu+\theta+\phi)(\mu+\theta+\sigma \phi+(1-\sigma) c \gamma)}{\sigma(1-\sigma) \phi}
\end{aligned}
$$

Therefore, the analogue of Theorem 1 is:

Theorem 4. For model (3), if $R(\phi)>1$, then the unique endemic equilibrium is globally asymptotically stable. If $R(\phi)<1$, then the disease-free equilibrium is globally stable unless

(i) $\beta>\beta_{\gamma a}$ and $\beta>\beta_{\gamma b}$, or, equivalently,

(ii) $(\mu+\theta+\sigma \phi)^{2}<(\mu+c) \sigma(1-\sigma) \phi-c \gamma(1-\sigma)(\mu+\theta+\sigma \phi)$ and $\beta>\beta_{\gamma b}$,

in which case there is a locally stable endemic equilibrium in competition with the locally stable disease-free equilibrium. 
We note, as did Hadeler and Castillo-Chávez, that if recovery brings permanent immunity, i.e., $\gamma=1$ and $\theta=0$, there can be no backward bifurcation; in particular the first condition in (ii) above is violated.

As a final remark, we note that Hadeler and van den Driessche consider a further generalized model in [23]; for example, if we let a proportion $\kappa, 0 \leq \kappa \leq 1$, of new arrivals into the population enter the vaccinated class, the expression for $R(\phi)$ is further reduced as follows: $R(\phi)=\frac{\beta}{\mu+c} \frac{\mu+\theta+\sigma \phi-(1-\sigma) \mu \kappa}{\mu+\theta+\phi}$.

\subsection{Disease-induced mortality}

Finally, we consider the case where the disease produces non-negligible death in the infected population. Model equations now give rise to a system where the total population is not constant:

$$
\begin{gathered}
S^{\prime}=k-\beta S I / N-(\mu+\phi) S+c I+\theta V \\
I^{\prime}=\beta(S+\sigma V) I / N-(\mu+d+c) I \\
V^{\prime}=\phi S-\sigma \beta V I / N-(\mu+\theta) V
\end{gathered}
$$

where $k$ is now the recruitment rate of susceptibles and $d$ is the disease-induced mortality. In this case the basic reproductive number stands as

$$
R_{0}=\frac{\beta}{\mu+c+d}
$$

The vaccination reproduction number can be computed as

$$
R(\phi)=R_{0} \frac{\mu+\theta+\sigma \phi}{\mu+\theta+\phi} .
$$

The introduction of disease-induced mortality in our model does not allow its reduction to a lower dimensional system like (1). Nevertheless the backward bifurcation phenomenon still exists for values of $R(\phi)<1$ as the following numerical example suggests (see Figure 4).

\section{Discussion}

Public health policies have consequences at the population level. In particular, the application of vaccines has to take into consideration not only vaccine-related parameters such as coverage, efficacy and so forth, but also social factors (risk behaviors) that may alter the expected theoretical predictions. In the introduction section we discussed several instances in which mathematical models help to identify plausible harmful effects of otherwise straightforward disease control strategies. The AIDS pandemic together with other emerging infectious diseases has underlined the necessity of careful control and planning of public health actions to prevent these effects that can go from spreading the diseases instead of eradicating it, to promoting the persistence of pathogen strains resistant to treatment drugs.

Vaccines that attempt to reduce susceptiblity to infection can be characterized by at least three important parameters represented in our model: coverage (represented by $\phi$ ), efficacy (represented by $\sigma$ ), and waning period (represented by $\theta$ ). Arguably not all of these are important in all vaccines. However, all of them interact and determine to a certain degree the success or failure of a vaccinaton program. Coverage and efficacy are related to costs either in terms of vaccination 


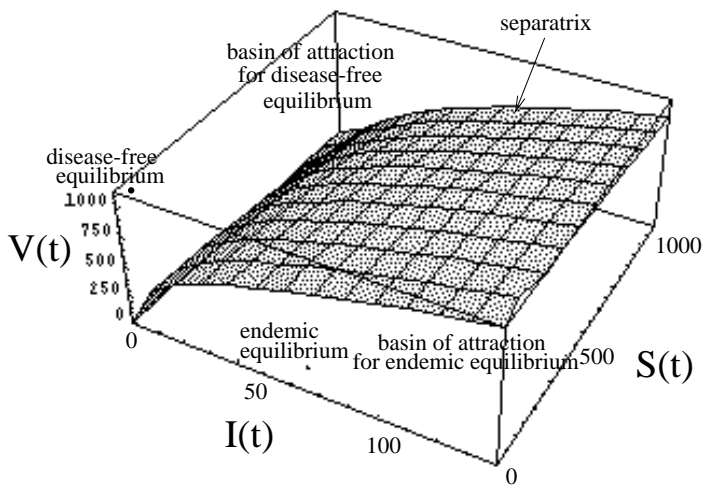

Figure 4: Multiple steady-states for the full 3D vaccination model (4)

for the parameter values $\mu=0.01, c=0.0, \theta=0.01, \sigma=.02$, $d=0.11, k=10, \beta=3.27$, and $0.9737<R(\phi)<1$.

The separatrix divides the state space into two basins of attraction, one for each of the two stable equilibria (shown).

campaigns, accessibility of vaccines, propaganda, etc., or in terms of intrinsic factors, e.g., quality of the vaccine.

The model and its generalization presented and analyzed in this paper is perhaps the simplest caricature possible of a vaccination program applied to a general population, and, in summary, it shows that a vaccination campaign $\phi$ meant to reduce a disease's reproduction number $R(\phi)$ below one may fail to control the disease:

- if the aim is to prevent an epidemic outbreak, a large initial number of infective persons can cause a high endemicity level to arise rather suddenly even if the vaccine-reduced reproduction number is below threshold.

- if the aim is to eradicate an already established disease, bringing the vaccine-reduced reproduction number below one may not be sufficient to do so.

Above, we have given a complete analysis of a simple model exhibiting a backward bifurcation - the simplest of which we are aware. Techniques focusing on the bifurcation(s) present have yielded local stability information in a situation where more traditional methods require difficult calculations; in this way the bifurcation analysis has allowed us to identify the parameters involved in this phenomenon.

Moreover, within the context of our models, we have seen that a partially effective vaccination program, in combination with a sufficiently high contact rate, can actually enable an invasion to succeed where it would normally die out. Keep in mind, however, that the vaccination program modeled in this paper does not, strictly speaking, make things worse: Without vaccination, the disease is guaranteed to die out if $\beta /(\mu+c)<1$, and (cf. Lemma 1) this remains true under vaccination. Rather, as discussed at the end of Section 2.2 , the vaccination program extends the local stability of the disease-free equilibrium in such a way that under certain conditions, an invasion of infectives can cause an endemic state to arise in a previously disease-free environment without any of the epidemiological parameters changing.

More quantitatively, we can answer the question of whether or not to vaccinate as follows. In general, a vaccination program is of interest when parameters are such that $R(\phi)<1<R_{0}$, i.e., without vaccination the disease will persist, but if everyone were vaccinated, the disease would 
normally die out. In such a situation, we wish to know how high the vaccination rate $\phi$ must be, not only to reduce $R(\phi)$ beneath 1 but to ensure that no endemic equilibria are possible. From the first inequality in (ii) of Theorem 4, we see that a backward bifurcation will be possible for some values of $\phi$ iff $Q \equiv 2(\mu+\theta)-(1-\sigma)[\mu+c(1-\gamma)]<0$. Since $\mu$ should be available from demographic data and $\theta, \sigma, c$ and $\gamma$ can be measured from controlled studies of the vaccine, this condition can first be determined. If the inequality above is obeyed, it is still possible to avoid multiple endemic equilibria by keeping $\phi$ out of the "danger zone" $\frac{1}{2 \sigma}\left[-Q \pm \sqrt{Q^{2}-4(\mu+\theta)(\mu+\theta+c \gamma(1-\sigma))}\right]$ (also obtained from (ii) in Theorem 4), or else by keeping the contact rate sufficiently low: $\beta<\beta_{\gamma b}$.

Third, when two at-risk populations interact, each population is capable of enabling an invasion to persist, regardless of the efforts of the other population. This suggests that such interacting populations, such as nearby border towns or other distinct groups whose geographical distribution overlaps, must include in their disease control policy a vaccination strategy that involves both groups - in other words, think globally.

The notion of vaccination in this context can also be extended to a more general description of education and behavior. In the context of a sexually transmitted disease, one might consider an "educated" class which employs practices that reduce the risk of infection. Hadeler and Müller discuss this notion with respect to two-sex models in [20]. In the context of infections communicable through direct contact, one could consider as "vaccinated" those individuals who conscientiously wash their hands, glassware, etc.

We could also consider these results in a metapopulation context - for example, an idea or philosophy spreading in a population for which we wish to determine what conditions are necessary for the idea to become accepted in the society under study. Cavalli-Sforza and Feldman [9] gave quantitative consideration of the way culture is transmitted in a population, and one might model the spread of ideas such as social reforms or new traditions in this way. Portes and Hao [43] also studied the loss of native languages across generations in families of immigrants to countries where another language is spoken - like English in the U.S. In these cases, a "vaccination program" would correspond to resistance to change, either organized or through individuals' negative experiences with the new idea. As an example, we might consider the kind of systemic changes currently recommended by national organizations in science and math education (see, e.g., [41]). The changes require a different approach to teaching which some teachers may view as challenging, threatening, or ineffective. We may consider two different populations of teachers, or one of teacher educators and one of teachers in schools, which resist or accept the new ideas at different levels and interact through classes, workshops, and more informal discussions. In this context, Theorem 3 says that not only will the idea not die out as long as one group pushes hard enough for it, but partial resistance to the idea may actually enable it to maintain a steady faction within both groups in circumstances where it would die out if ignored (no "vaccination program"). This conclusion supports the observation that attempts to censor or decry a given phenomenon frequently result in calling greater attention to it, with the result that some small fraction take it up as a cause or incorporate it into their lives.

We may also speculate that similar results will occur for more than two interacting populations; the more general case is an area for future study, as is the more explicit consideration of the non-epidemiological contexts suggested above when formulating the model. Finally, also worthy of further study (and addressed in part in [23] and [37]) is the application of the bifurcationrelated techniques illustrated in this paper to obtain a more complete, cohesive idea of what causes backward bifurcations in epidemic models, a phenomenon with important biological consequences. 


\section{Appendix. Proofs}

Lemma 1. For the system (1), the disease-free equilibrium is globally asymptotically stable if $R(0)=R_{0}<1$.

Proof. We first rewrite the system as a set of integral equations:

$$
\begin{gathered}
I(t)=\int_{-\infty}^{t}(N-I(s)-(1-\sigma) V(s)) \beta \frac{I(s)}{N} e^{-(\mu+c)(t-s)} d s \\
V(t)=\int_{-\infty}^{t}\left(\phi(N-I(s)-V(s))-\beta \sigma V(s) \frac{I(s)}{N}\right) e^{-(\mu+\theta)(t-s)} d s
\end{gathered}
$$

We now use the substitution $x=t-s$, take the lim sup of both sides of the equation for $I(t)$ and apply the fact that $\lim \sup \int f \leq \int \lim \sup f$ (see Lemma 2 in [35]) to get

$$
\begin{aligned}
\limsup _{t \rightarrow \infty} I(t) & =\limsup _{t \rightarrow \infty} \int_{0}^{\infty}(N-I(t-x)-(1-\sigma) V(t-x)) \beta \frac{I(t-x)}{N} e^{-(\mu+c) x} d x \\
& \leq \int_{0}^{\infty} \limsup _{t \rightarrow \infty}(N-I(t-x)-(1-\sigma) V(t-x)) \beta \limsup _{t \rightarrow \infty} \frac{I(t-x)}{N} e^{-(\mu+c) x} d x \\
& \leq \limsup _{t \rightarrow \infty}(N-I(t)-(1-\sigma) V(t)) \beta \limsup _{t \rightarrow \infty} \frac{I(t)}{N} \int_{0}^{\infty} e^{-(\mu+c) x} d x \\
& \leq N \cdot \beta\left(\limsup _{t \rightarrow \infty} I(t)\right) / N \frac{1}{\mu+c} \\
& =\frac{\beta}{\mu+c} \limsup _{t \rightarrow \infty} I(t)
\end{aligned}
$$

Thus if $\frac{\beta}{\mu+c}<1$, we have the strict inequality (and contradiction) $\limsup _{t \rightarrow \infty} I(t)<\limsup _{t \rightarrow \infty} I(t)$, unless $\lim \sup _{t \rightarrow \infty} I(t)=0$.

Theorem 2. For system 2 , the disease-free equilibrium is globally asymptotically stable if

$$
R_{0}=\frac{\beta_{1}}{(\mu+c)} \frac{N_{1}}{N}+\frac{\beta_{2}}{(\mu+c)} \frac{N_{2}}{N}<1 .
$$

Proof. By rewriting the system as a set of integral equations and applying the methods of Theorem 1, we can obtain the inequalities

$$
\begin{aligned}
& \limsup _{t \rightarrow \infty} I_{1}(t) \leq \frac{N_{1}}{N}\left(\frac{\beta_{1}}{\mu+c} \limsup _{t \rightarrow \infty} I_{1}(t)+\frac{\beta_{2}}{\mu+c} \limsup _{t \rightarrow \infty} I_{2}(t)\right), \\
& \limsup _{t \rightarrow \infty} I_{2}(t) \leq \frac{N_{2}}{N}\left(\frac{\beta_{1}}{\mu+c} \limsup _{t \rightarrow \infty} I_{1}(t)+\frac{\beta_{2}}{\mu+c} \limsup _{t \rightarrow \infty} I_{2}(t)\right) .
\end{aligned}
$$

We now apply Lemma 3 from [35] to get that if $R_{0}<1$, then $\lim \sup _{t \rightarrow \infty} I_{1}(t)=\lim \sup _{t \rightarrow \infty} I_{2}(t)=$ 0 .

Theorem 3. For model (2), with $R(\Phi)$ as defined above, 
(i) for $R(\Phi)<1$, there is an even number of endemic equilibria;

(ii) for $R(\Phi)>1$, there is an odd number of endemic equilibria;

(iii) for $R(\Phi)<1$, either of the conditions $\beta_{1}>\max \left(\beta_{1 a}, \beta_{1 b}\right), \beta_{2}>\max \left(\beta_{2 a}, \beta_{2 b}\right)$ is sufficient (but not necessary) to cause two endemic equilibria,

where $\beta_{1 a} \equiv \frac{N}{N_{1}}\left(\mu+c+\frac{\mu+\theta_{1}+\sigma_{1} \phi_{1}}{\sigma_{1}}\right), \beta_{1 b} \equiv \frac{N}{N_{1}}\left(\mu+c-\frac{\mu+\theta_{1}+\sigma_{1} \phi_{1}}{\sigma_{1}}+\frac{2}{\sigma_{1}} \sqrt{(\mu+c) \sigma_{1}\left(1-\sigma_{1}\right) \phi_{1}}\right)$, and $\beta_{2 a}$ and $\beta_{2 b}$ are defined similarly.

Proof. The equilibrium conditions can be written $h_{1}(x, y)=0$ and $h_{2}(x, y)=0$, where

$$
\begin{aligned}
& h_{1}(x, y)=a_{10} x+a_{20} x^{2}-a_{30} x^{3}+a_{01} y+a_{11} x y-a_{21} x^{2} y+a_{02} y^{2}-a_{12} x y^{2} \\
& h_{2}(x, y)=b_{10} y+b_{20} y^{2}-b_{30} y^{3}+b_{01} x+b_{11} x y-b_{21} x y^{2}+b_{02} x^{2}-b_{12} x^{2} y
\end{aligned}
$$

and the $a_{i j}$ and $b_{i j}$ are certain functions of the model parameters. Our proof will therefore consider intersections of the zero-contours of these two functions. The argument is technical, and not necessary for a first reading. The accompanying figures 5 and 6 may provide some intuition.

Let us consider $h_{1}$ and $h_{2}$ on the boundary of the domain. For $y$ in $\left[0, N_{2} / N\right]$,

$$
\begin{gathered}
h_{1}(0, y)=\beta_{2}\left(N_{1} / N\right) y\left(\beta_{2} \sigma_{1} y+\mu+\theta_{1}+\sigma_{1} \phi_{1}\right) \geq 0, \\
h_{1}\left(\frac{N_{1}}{N}, y\right)=-(\mu+c) N_{1}\left(\beta_{1} \sigma_{1} N_{1}+\left(\mu+\theta_{1}+\phi_{1}+\beta_{2} \sigma_{1} y\right) N\right) / N^{2} \leq 0,
\end{gathered}
$$

so that $h_{1}$ is zero at the origin (which corresponds to the disease-free equilibrium), strictly positive along the rest of the left side, and strictly negative along the right side. Thus some zero-contour of $h_{1}$ must exit the domain through the top side of the domain, either one or three times, and likewise some zero-contour of $h_{1}$ must enter the domain either through the origin or through the bottom side, either one or three times. This forces at least one to cross all the way from bottom to top.

Furthermore, the gradient at the origin is

$$
\nabla h_{1}(0,0)=(\mu+c)\left(\mu+\theta_{1}+\phi_{1}\right)\left\{-1+R_{1}\left(\phi_{1}\right) \frac{N_{1}}{N}, \frac{\beta_{2}}{\beta_{1}} R_{1}\left(\phi_{1}\right) \frac{N_{1}}{N}\right\}
$$

so the slope at the origin of $h_{1}$ 's zero-contour, which is perpendicular to the gradient, is $\frac{\beta_{1}}{\beta_{2}}\left(\frac{1}{R_{1}\left(\phi_{1}\right) \frac{N_{1}}{N}}-1\right)$. Thus this slope is positive, and the zero-contour enters the domain through the origin, precisely when $R_{1}(\phi) \frac{N_{1}}{N}<1$. Otherwise, the slope is negative, so the zero-contour passes through the second and fourth quadrants (instead of entering the first quadrant at the origin), and $h_{1}$ is positive to the immediate right of the origin, so that the zero-contour must enter the domain somewhere along the bottom side.

We can establish similar conditions on $h_{2}$, and find that $h_{2}$ is zero at the origin, strictly positive along the rest of the bottom side, and strictly negative along the top side. When $R_{2}\left(\phi_{2}\right) \frac{N_{2}}{N}<1$, $h_{2}$ 's zero-contour enters the domain through the origin and exits through the right side. When $R_{2}\left(\phi_{2}\right) \frac{N_{2}}{N}>1$, it enters not through the origin but through the left side, also to exit through the right side.

Let us now consider three cases:

1. $R_{1}\left(\phi_{1}\right) \frac{N_{1}}{N}>1$ or $R_{2}\left(\phi_{2}\right) \frac{N_{2}}{N}>1$ (and, consequently, $R(\Phi)>1$ );

2. $R_{1}\left(\phi_{1}\right) \frac{N_{1}}{N}<1$ and $R_{2}\left(\phi_{2}\right) \frac{N_{2}}{N}<1$ but $R(\Phi)>1$;

3. $R_{1}\left(\phi_{1}\right) \frac{N_{1}}{N}<1, R_{2}\left(\phi_{2}\right) \frac{N_{2}}{N}<1$ and $R(\Phi)<1$. 


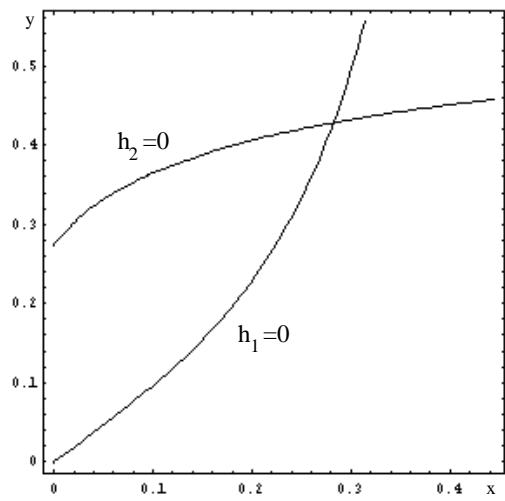

(a) $R_{1}\left(\phi_{1}\right) \frac{N_{1}}{N}<1$, $R_{2}\left(\phi_{2}\right) \frac{N_{2}}{N}>1$

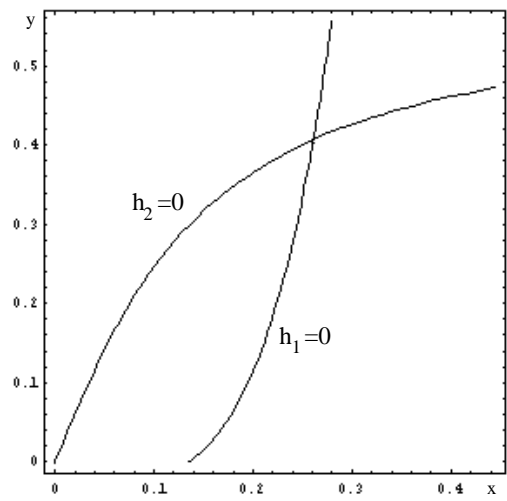

(b) $R_{1}\left(\phi_{1}\right) \frac{N_{1}}{N}>1$, $R_{2}\left(\phi_{2}\right) \frac{N_{2}}{N}<1$

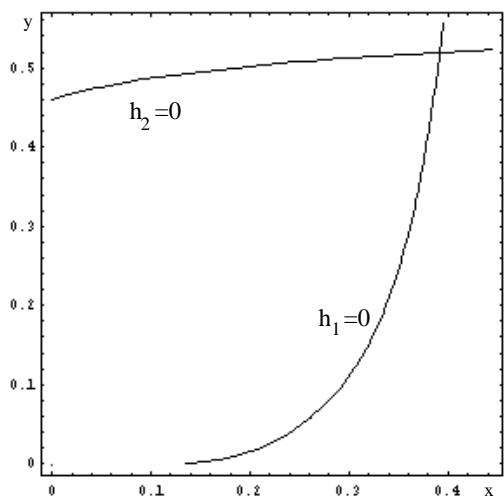

(c) $R_{1}\left(\phi_{1}\right) \frac{N_{1}}{N}>1$, $R_{2}\left(\phi_{2}\right) \frac{N_{2}}{N}>1$

Figure 5: Intersecting zero contours of $h_{1}$ and $h_{2}$ for Case 1: $R_{1}\left(\phi_{1}\right) \frac{N_{1}}{N}>1$ or $R_{2}\left(\phi_{2}\right) \frac{N_{2}}{N}>1$

CASE 1. When either $R_{1}\left(\phi_{1}\right) \frac{N_{1}}{N}>1$ or $R_{2}\left(\phi_{2}\right) \frac{N_{2}}{N}>1$, there must be an endemic equilibrium, by virtue of the fact that at least one zero contour crosses from side to opposite side, obliging the other to cross it. Figure 5 illustrates the three general possibilities in this case.

CASE 2. If both $R_{1}\left(\phi_{1}\right) \frac{N_{1}}{N}<1$ and $R_{2}\left(\phi_{2}\right) \frac{N_{2}}{N}<1$, both zero-contours enter the domain at the origin. Whether or not they intersect depends in part upon the slopes of those zero-contours there. The slope of $h_{1}$ 's zero-contour at the origin, as calculated above, is $\frac{\beta_{1}}{\beta_{2}}\left(\frac{1}{R_{1}\left(\phi_{1}\right) \frac{N_{1}}{N}}-1\right)$. The slope of $h_{2}$ 's zero-contour at $(0,0)$ is $\frac{\beta_{1}}{\beta_{2}}\left(\frac{1}{\frac{1}{R_{2}\left(\phi_{2}\right) \frac{N_{2}}{N}}-1}\right)$. Thus we can calculate the ratio of the first to the second; this is $K \equiv\left(\frac{1}{R_{1}\left(\phi_{1}\right) \frac{N_{1}}{N}}-1\right)\left(\frac{1}{R_{2}\left(\phi_{2}\right) \frac{N_{2}}{N}}-1\right)$. When this ratio is less than one, $h_{1}$ 's zero-contour starts out below $h_{2}$ 's, so again they must intersect, since we know $h_{1}$ 's zero-contour ends up above $h_{2}$ 's. But the condition $K<1$ is equivalent to $R(\Phi)>1$. Thus when $R_{1}(\phi) \frac{N_{1}}{N}<1$ and $R_{2}(\phi) \frac{N_{2}}{N}<1$, but $R(\Phi)>1$, we must again have one (or three) endemic equilibrium.

Now note that Cases 1 and 2 combine to form statement (ii) of the theorem, since if either $R_{1}(\phi) \frac{N_{1}}{N}>1$ or $R_{2}(\phi) \frac{N_{2}}{N}>1$, then automatically $R(\Phi)>1$, since the former two left-hand sides add to make $R(\Phi)$. Hence anytime $R(\Phi)>1$, we must have one endemic equilibrium (or three).

CASE 3. The next question is whether or not we can have endemic equilibria when $R(\Phi)<1$. Since $h_{1}$ 's zero-contour both starts and ends above $h_{2}$ 's, there should be an even number of them in any case, except at a bifurcation point. This proves statement (i) of the theorem.

In fact, it is possible to have either zero or two endemic equilibria. Figure 6 illustrates the three possibilities that may occur when both $R_{1}\left(\phi_{1}\right) \frac{N_{1}}{N}$ and $R_{2}\left(\phi_{2}\right) \frac{N_{2}}{N}$ are less than 1 . Figure 7 gives a plot of $h_{1}(x, x)$ corresponding to the parameter values used to generate Figure 6 (b), which, being symmetric, give $h_{1}(x, y)=h_{2}(y, x)$. In this way we can see that the zero contours do indeed cross twice away from the origin, a fact which may not be clear from Figure 6(b) (which corresponds to statement (iii) of the theorem).

Let us now consider $h_{1}(x, 0)=a_{10} x+a_{20} x^{2}-a_{30} x^{3}$. If we separate the factor of $x$, what remains is identical to the quadratic $f(x)$ defined in Section 2.2, so we know that, for $\beta_{10}, \beta_{1 a}, \beta_{1 b}$ defined analogously (and each having a factor of $\frac{N}{N_{1}}$ in front), the number of times $h_{1}$ 's zero-contour crosses the bottom of the domain (origin excluded) is one if $\beta_{1}>\beta_{10}$, two if $\max \left(\beta_{1 a}, \beta_{1 b}\right)<\beta_{1}<\beta_{10}$, and 


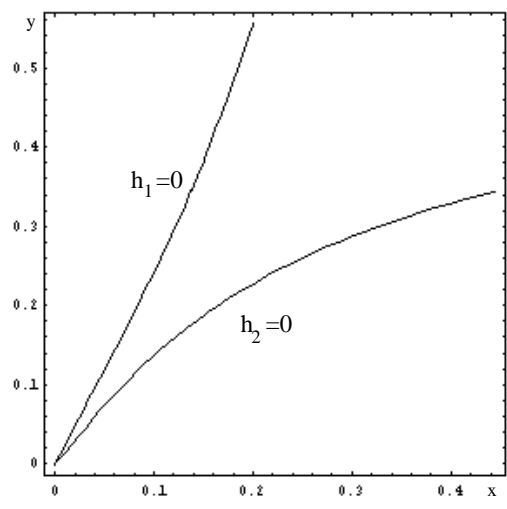

(a) $R(\Phi)<1$, no endemic equilibria (Case 3)

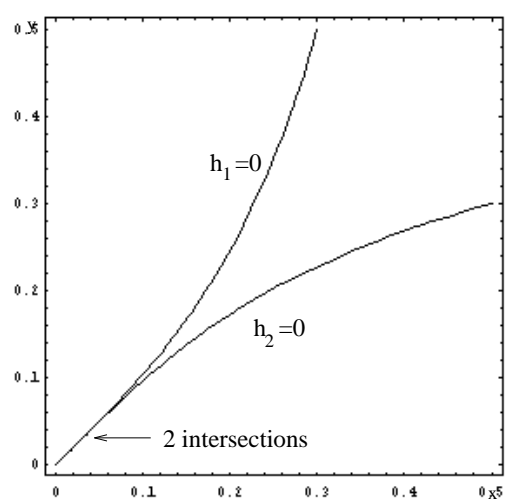

(b) $R(\Phi)<1$, two endemic equilibria (Case 3)

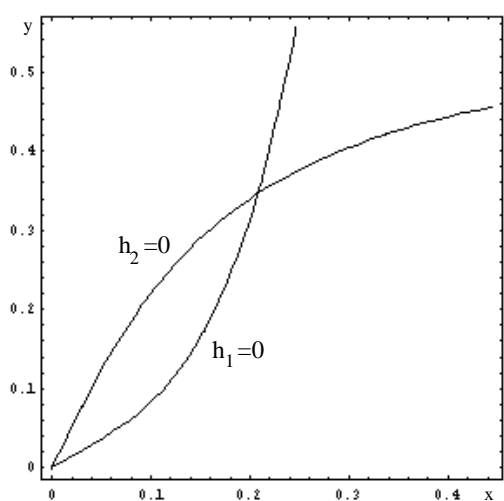

(c) $R(\Phi)>1$ one endemic equilibrium (Case 2)

Figure 6: Intersecting zero contours of $h_{1}$ and $h_{2}$ for $R_{1}\left(\phi_{1}\right) \frac{N_{1}}{N}<1$ and $R_{2}\left(\phi_{2}\right) \frac{N_{2}}{N}<1$

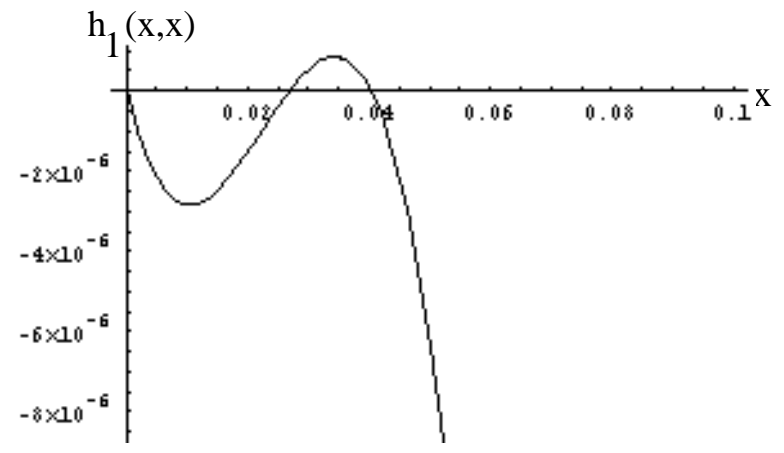

Figure 7: Graph of $h_{1}(x, x)$ corresponding to Figure 6(b) 


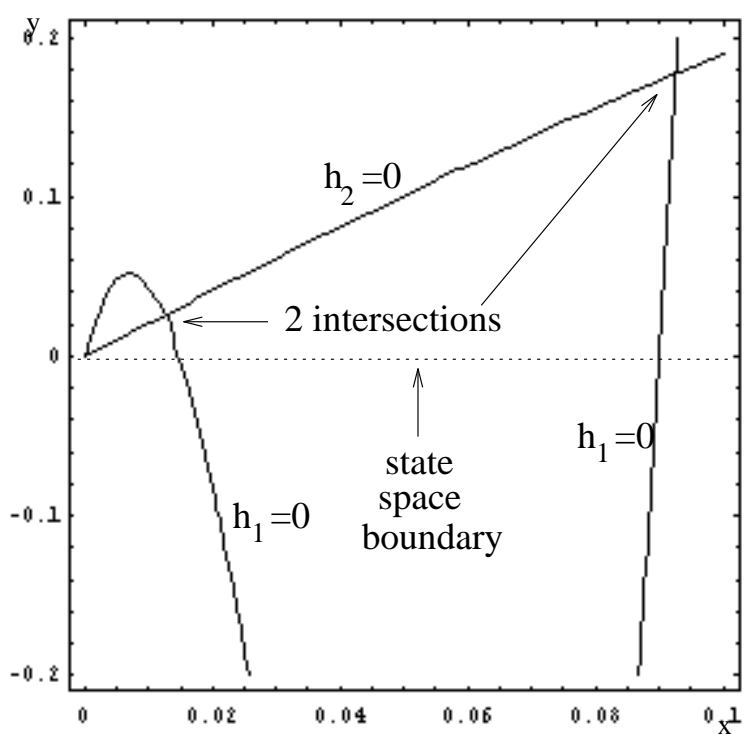

Figure 8: Zero-contours of $h_{1}$ and $h_{2}$ intersecting twice under the conditions $R(\phi)<1$ and $\beta_{1}>\max \left(\beta_{1 a}, \beta_{1 b}\right)$

zero otherwise. (Again we exclude the degenerate case $\beta_{1 b}=\beta_{1}<\beta_{10}$, where the number is one.)

In the case where the zero contour crosses twice more, we can verify that it crosses down from inside the domain and then re-enters the domain from below by calculating the zero-contour's concavity (it is positive) at the degenerate case cited above. Thus if $R(\phi)<1$, the condition $\beta_{1}>\max \left(\beta_{1 a}, \beta_{1 b}\right)$ is sufficient (but not necessary) to guarantee that the zero-contours of $h_{1}$ and $h_{2}$ intersect twice (see Figure 8). The same therefore holds with respect to $h_{2}$ and the condition $\beta_{2}>\max \left(\beta_{2 a}, \beta_{2 b}\right)$. This shows that either one of the two interacting populations has the capability to cause a backward bifurcation, and endemic equilibria, regardless of the efforts of the other population. 
Discussions with Carlos M. Hernández helped to clarify several aspects of vaccination campaigns. JXVH acknowledges support from a CONACYT grant 1998 and UAM-I internal grant. CMKZ research was partially supported by an REP grant from the University of Texas at Arlington during the summer of 1998.

\section{References}

[1] R. M. Anderson and R. M. May, Vaccination and herd immunity to infectious diseases. Nature 318: 323-329. (1985).

[2] N.G. Becker and D.N. Starczak, Optimal vaccination strategies for a community of households. Mathematical Biosciences 139: 117-132. (1997).

[3] S.M. Blower and J.L. Gerberding, Understanding, predicting and controlling the emergence of drug-resistant tuberculosis: a theoretical framework. Journal of Molecular Medicine 76: 624-636. (1998).

[4] S.M. Blower and A.R. McLean, Prophylactic vaccines, risk behavior change, and the probability of eradicating HIV in San Francisco. Science 265: 1451-1454. (1994).

[5] S.M. Blower, P.M. Small and P.C. Hopewell, Control stategies for tuberculosis epidemics: new models for old problems. Science 273: 497-500. (1996).

[6] S. Busenberg and P. van den Driessche, Analysis of a disease transmission model in a population with varying size. J. Math. Biol. 28: 257-270. (1990).

[7] J. Carr, Applications of centre manifold theory, Applied Mathematical Sciences, 35, SpringerVerlag, New York, 1981.

[8] C. Castillo-Chávez, K. L. Cooke, W. Huang and J. Li, On the role of long incubation periods in the dynamics of AIDS. Part II: Multiple-group models, in Mathematical and statistical approaches to AIDS epidemiology, C. Castillo-Chávez, ed., Lecture Notes in Biomathematics 83, Springer-Verlag, New York, 1989, pp. 200-217.

[9] L. L. Cavalli-Sforza, M. W. Feldman, Cultural transmission and evolution: a quantitative approach, Monographs in Population Biology 16, Princeton University Press, Princeton, NJ, 1981.

[10] O. Diekmann, K. Dietz and J. A. P. Heesterbeek, The basic reproductive ratio for sexually transmitted diseases: I. Theoretical considerations. Math. Biosci. 107: 325-339. (1991).

[11] O. Diekmann, J. A. P. Heesterbeek and J. A. J. Metz, On the definition and the computation of the basic reproduction ratio $R_{0}$ in models for infectious diseases in heterogeneous populations. J. Math. Biol. 28: 365-382. (1990).

[12] J. Dushoff, Incorporating immunological ideas in epidemiological models. J. Theoretical Biol. 180: 181-187. (1996).

[13] J. Dushoff, W. Huang and C. Castillo-Chávez, Backwards bifurcations and catastrophe in simple models of fatal diseases. J. Math. Biol. 36: 227-248. (1998). 
[14] D. Greenhalgh, Vaccination campaigns for common childhood diseases. Math. Biosci. 100: 201-240. (1990).

[15] M. Gyllenberg, I. Hanski and A. Hastings, Structured metapopulation models. In Metapopulation Biology, I. Hanski and M.E. Gilpin, eds. Academic Press, San Diego (1997). pp. 93-122.

[16] M. Haber, I.M. Longini and M.E. Halloran, Measures of the effects of vaccination in a randomly mixing population. International Journal of Epidemiology 20: 300-310. (1991).

[17] M. Haber, I.M. Longini and M.E. Halloran, Estimation of vaccine efficacy in outbreaks of acute infectious diseases. Statistics in Medicine 10: 1573-1584. (1991).

[18] K. P. Hadeler, Models for endemic diseases, Mathematics in biology and medicine, V. Capasso, E. Grosso and S. L. Paveri-Fontana, eds., Lecture Notes in Biomathematics 57, Springer-Verlag, New York, 1985, pp. 127-134.

[19] K. P. Hadeler and C. Castillo-Chávez, A core group model for disease transmission. Math. Biosci. 128: 41-55. (1995).

[20] K. P. Hadeler and J. Müller, The effects of vaccination on sexually transmitted disease in heterosexual populations, in Mathematical population dynamics, O. Arino, D. Axelrod, M. Kimmel and M. Langlois, eds., Third International Conference, Vol. 1, Marcel Dekker, Winnipeg, 1995, pp. 251-278.

[21] K. P. Hadeler and J. Müller, Optimal vaccination patterns in age-structured populations I: the reproduction number. In Models for Infectious Human Diseases: Their Structure and Relation to Data, V. Isham and G. Medley, eds., Cambridge University Press, Cambridge, 1996.

[22] K. P. Hadeler and J. Müller, Optimal vaccination patterns in age-structured populations II: optimal strategies. In Models for Infectious Human Diseases: Their Structure and Relation to Data, V. Isham and G. Medley, eds., Cambridge University Press, Cambridge, 1996.

[23] K. P. Hadeler and P. van den Driessche, Backward bifurcation in epidemic control. Math. Biosci. 146: 15-35. (1997).

[24] W. Hahn, Stability of motion, Springer-Verlag, New York, 1967.

[25] M.E. Halloran, Discussion: Vaccine effect on susceptibility. Statistics in Medicine 15: 24052412. (1996).

[26] H. W. Hethcote, Qualitative analyses for communicable disease models. Math. Biosci. 28: 335-356. (1976).

[27] H. W. Hethcote, Three basic epidemiological models, in S. A. Levin, T. G. Hallam and L. J. Gross (Eds.), Applied mathematical ecology (Trieste, 1986), Biomathematics, 18, SpringerVerlag, New York, 1989, pp. 119-144.

[28] H.W. Hethcote, Optimal ages of vaccination for measles. Mathematical Biosciences 89: 29-52. (1988).

[29] H.W. Hethcote and J.W. van Ark, Epidemiological models for heterogeneous populations: proportionate mixing, parameter estimation and immunization programs. Mathematical Biosciences 84: 85-118. (1987). 
[30] Y.H. Hsieh and J.X. Velasco-Hernández. Community treatment of HIV-1: initial stage and asymptotic dynamics. BioSystems 35: 75-81. (1995).

[31] W. Huang, K. L. Cooke and C. Castillo-Chávez, Stability and bifurcation for a multiple-group model for the dynamics of HIV/AIDS transmission. SIAM J. Appl. Math 52: 835-854. (1992).

[32] J.M. Hyman and J. Li, Behavior changes in SIS STD models with selective mixing. SIAM Journal of Applied Mathematics 57: 1082-1094. (1997).

[33] J.M. Hyman and J. Li, Modeling the effectiveness of isolation strategies in preventing STD epidemics. SIAM Journal of Applied Mathematics 58: 912-925. (1998).

[34] C. M. Kribs, Core recruitment effects in modeling a sexually transmitted disease, doctoral dissertation, University of Wisconsin, 1997.

[35] C. M. Kribs-Zaleta, Structured models for heterosexual disease transmission. Math. Biosci. 160(1): 83-108 (1999).

[36] C. M. Kribs-Zaleta, Core recruitment effects in SIS models with constant total populations. Math. Biosci. 160(2): 109-158 (1999).

[37] C. M. Kribs-Zaleta, Center manifolds and normal forms in epidemic models, to appear.

[38] A. Lajmanovich and J.A. Yorke, A deterministic model for gonorrhea in a nonhomogeneous population. Math. Biosci. 28: 221-236 (1976).

[39] R. K. Miller, Nonlinear Volterra integral equations, W. A. Benjamin, Inc., New York, 1971.

[40] J. Müller, Optimal vaccination strategies-for whom? Mathematical Biosciences 139: 133-154. (1997).

[41] National Research Council, Everybody counts - A report to the nation on the future of mathematics education, National Academy Press, Washington, DC, 1989.

[42] T.C. Porco and S. M. Blower, Designing HIV vaccination policies: subtypes and crossimmunity. Interfaces 28: 167-190. (1998).

[43] A. Portes and L. Hao, E Pluribus Unum: Bilingualism and language loss in the second generation. Presented at the symposium on the second generation, sponsored by the Jerome Levy Economics Institute of Bard College, October 23-25, 1997. http://econwpa.wustl.edu:8089/eps/mac/papers/9805/9805006.pdf

[44] J.X. Velasco-Hernández and Y.H. Hsieh. Modeling the effect of treatment and behavioral change in HIV transmission dynamics. Journal of Mathematical Biology 32: 233-249. (1994).

[45] J.X. Velasco-Hernández, F. Brauer and C. Castillo-Chávez, Effects of treatment and prevalence-dependent recruitment on the dynamics of a fatal disease. IMA Journal of Mathematics Applied to Medicine and Biology 13: 175-192. (1996). 